$$
\text { "gyongyosi" — 2004/7/23 — 13:57 — page } 15-\# 1
$$

\title{
Using the computer to visualise graph-oriented problems
}

\author{
ERIKA GYÖNGYÖSI
}

Abstract. The computer, if used more effectively, could bring advances that would improve mathematical education dramatically, not least with its ability to calculate quickly and display moving graphics. There is a gap between research results of the enthusiastic innovators in the field of information technology and the current weak integration of the use of computers into mathematics teaching.

This paper examines what exactly the real potentials of using some mathematics computer software are to support mathematics teaching and learning in graph-oriented problems, more specifically we try to estimate the value added impact of computer use in the mathematics learning process.

While electronic computation has been used by mathematicians for five decades, it has been in the hands of teachers and learners for at most three decades but the real breakthrough of decentralised and personalised micro-computer-based computing has been widely available for less than two decades. And it is the latter facility that has brought the greatest promise for computers in mathematics education. That computational aids overall do a better job of holding students' mathematical interest and challenging them to use their intellectual power to mathematical achievement than do traditional static media is unquestionable. The real question needing investigation concerns the circumstances where each is appropriate.

A case study enabled a specification of advantages and obstacles of using computers in graph-oriented questions. Individual students' interviews revealed two less able students' reactions, difficulties and misinterpretations while using computers in mathematics learning.

Among research outcomes is that the mathematical achievement of the two students observed improved and this makes teaching with computers an overriding priority for each defined teaching method.

This paper may not have been realised without the valuable help of the Hungarian Eötvös State Grant.

Copyright (C) 2004 by University of Debrecen 


$$
\text { "gyongyosi" — 2004/7/23 — 13:57 — page } 16 \text { — \#2 }
$$

Key words and phrases: computers, mathematics software, visualisation, process, procept, graph-oriented problems, improve.

ZDM Subject Classification: D34, D74, I24, H34, U54, U64.

\section{Introduction}

The visualisation used by students when learning mathematical concepts has been studied over a number of years and the increasing use of computers in mathematics and associated disciplines enables dynamic representations such as moving pictures, to provide cognitive support for greater potential.

Standard pictures found in text-books could lead to restricted development of mathematical concepts as they are static, and thus fail to fully convey the dynamic nature of many of the concepts, and they also tend to be limited in variety, leading to a restricted concept image being developed from only a few examples.

For instance, some students at secondary level could not link algebraic and graphical methods for solving quadratic equations. Thus whenever they have to solve a quadratic equation with negative discriminant graphically they get confused because they do not believe that the function exists at all. Even if students draw graphs of (say) $y=x^{2}+1$ they usually do not link the graphical solution of the secondary equation $0=x^{2}+1$ and the concept of the determinant of it. However, if they are able to interact with a computer programme that gives a graphical solution or an equation with negative discriminant, the phenomenon takes on a genuine meaning: "of course even if the quadratic equation has no zeros: we can see the graph representing it."

Unfortunately in the Hungarian curriculum the topic of functions is not included in algebra, however, in mathematics books it is an independent chapter together with sequences that makes it even more difficult for students to find relations between equations and functions. Thus graphical solutions are rarely given and neglected.

During my teaching practice I often observed that students in the case of complex problems such as solving difficult quadratic inequalities waste a lot of time and energy in the process of calculating the roots of a quadratic equation with the formula and they can not see the links between the concepts of roots and the zeros of the corresponding quadratic function not even between the zeros and the intersection points of the graph of the function and the $x$-axis. For example, if students have to solve the inequality $x^{3}+3>0$ they try to apply the formula 


$$
\text { "gyongyosi" — 2004/7/23 - 13:57 — page } 17 \text { — \#3 }
$$

to solve the quadratic equation $0=x^{3}+3$ and as there is a negative number under the root sign in the formula they conclude that there is no solution for the inequality. Moreover, many students get confused if the teacher asks them to draw the graph of a function that is always positive/negative.

As computers carry out the process of calculations students can concentrate on the overall patterns and continuity of the entire activity, thus the procedure itself becomes an entity and students may go on analysing relations between different mathematical concepts.

In Hungary there is a need for improving curriculum and changing teaching styles in order to develop learning approaches focused on relational understanding of mathematical concepts.

However, computer-based education can not solve every problem in education and as Kaput [6] concluded although many enthusiastic claims have been made for the positive impact of computers on the teaching and learning of mathematics, systematic evaluations of their impact are harder to come by.

Kaput and Thompson [6], [7] further warn that the uncritical acceptance of technological inventions created for other audiences do not fulfil expectations. Studies on computer-based teaching of mathematics do not help much to specify all of those learning difficulties in which the application of computers proves to be effective or ineffective and the extent to which they may contribute to the improvement of students' performance.

Our specific research objective is to estimate the 'value added' impact of the computer based teaching programme related to transformations of functions, solving quadratic equations and inequalities in terms of mathematical performance.

\section{Theoretical background}

Already Piaget $[11$, p. 49] has pointed out that "actions and operations become thematized objects of thought or assimilation". According to Meissner [10] this idea has become very important today to understand the development of concept images ${ }^{1}$ (Vorstellungen) in mathematics education as a process of "interiorization or reification or encapsulation". Vorstellungen are personal internal representations evoked by "Darstellungen" that is external representations

${ }^{1}$ Concept image is the name of a concept (notion) associated global cognitive structure, which contains the visual representations (pictures, diagrams, graphs) internal connections, concrete experiences, examples, characteristics, procedures 
of mathematical ideas. Computers are a mean of external representations and internal representations considerably depend on external representations.

According to Gray and Tall [3, p. 72] there is a "duality between process and concept in mathematics, in particular using the same symbolism to present both a process (such as the addition of two numbers $3+2$ ) and the product of that process (the sum $3+2$ ). The ambiguity of notation allows the successful thinker the flexibility in thought to move between the process to carry carry out a mathematical task and the concept to be mentally manipulated as part of a wider schema". The successful mathematical thinker uses a mental structure called procept [11, p. 251], "which is an amalgam of process and concept."

Gray and Tall [4] proposed the following definitions: "An elementary procept is the amalgam of three components: a process which produces a mathematical object, and a symbol which is used to represent either process or object. A procept consists a collection of elementary procepts which have the same object". In [15] we find examples for symbols as process and concept. For more details on the theory of procepts see Gray and Tall [3], Sfard [12], Dubinsky [2], Meissner [10] and others.

One of the fundamental ideas of mathematics is the concept of functions. To understand the concept of functions it is essential to comprehend the variables, rule and function values as a unity, that is as a procept. Sfard [13, p. 64] identified a constant three-step pattern in the successive transitions from operational to structural conceptions. "First there must be a process performed on the already familiar objects, then the idea of turning this process into a more compact, selfcontained whole should emerge, and finally an ability to view this new entity as a permanent object in its own right must be acquired."

In the case of functions the first step is a process during which concrete values are given as function variables and function values are calculated according to a given rule. Functions must be experienced through examples and counterexamples. Moreover, since calculators and computers reduce the burden of computing we can turn our attentions to study the relations between different variables, we can analyse properties, we can draw graphs. Thus, the idea of function turns into a more self-contained entity and the symbolism representing the rule between variables and function values tends to present not only the process of calculating function values but also the product of that process (the function itself). Finally, an ability to give the rule of a function from its graphical representation means the ability to view the function as a permanent object. Many students can 


$$
\text { "gyongyosi" — 2004/7/23 — 13:57 — page } 19-\# 5
$$

carry out easily the process of calculating function values of given variables and vice-versa but they do not view function as an object, however, it is essential to understand the concept of reverse functions.

Skemp (1978) distinguished between instrumental understanding and relational understanding. Instrumental understanding is characterized by selecting and applying appropriate rules to solve the problem without knowing why ("rules without reasons"). Only a specific Darstellung, an external observable behaviour, is expected: "Tell me what to do and I will do so". While relational understanding means to know exactly why certain rules are appropriate to use to solve the problem (precise description of reasons to rules). There is necessarily an adequate mathematical concept image as well as relations between other concepts behind.

An instrumental understanding is necessary but not sufficient to get the correct solution. From this point of view many manuals for calculators or computers or software packages only provoke the development of an instrumental (instrumental in terms of the 'machine') understanding. During relational understanding the meaning of the problem situation is in the foreground and the machine only is an aid to reduce the burden of sophisticated calculations or drawings or it is an aid to visualise relations or properties. There are many examples how calculators or computers can be used to improve relational understanding, starting with the work from David Tall on calculus. Many semantic activities are common today's classroom: using dynamic geometry software (Cabri, Euklides, and others), investigations with computer algebra software, using appropriate software-hardware configurations.

But till now there appear to be no reported general theories how calculators or computers can be used to improve relational understanding.

Though by no means a total solution, it is hoped that interactive work on the computer can give a better insight into the characteristics of functions that is potentially more meaningful.

Our hypothesis is that computers can be used to enhance learning mathematics, by reducing the burden of long and exhausting calculations or drawings and by visualizing relations or properties. The literature concerning the use of computers in education attributes to it a number of advantages, the majority of which are not specific to any particular software. More details on the advantages and challenges of using computers to improve mathematics education can be seen in [5]. More specifically the literature in this area ([1]) shows that the use of Computer Algebraic Systems is most frequently presented as: permitting a more 


$$
\text { "gyongyosi" — 2004/7/23 — 13:57 — page } 20-\# 6
$$

effective development of an experimental approach to mathematics, allowing for the exploration of problems which are more interesting than those usually encountered, and not strictly within the school syllabus, through using calculation aids (numerical, algebraic and graphical) provided by the computer and providing a more user-friendly atmosphere for teaching and more suited to the needs of the particular learner.

According to Tall [16, p. 2] "the computer is programmed to enable the user to manipulate examples of mathematical processes and to see them dynamically. Through experience in this way, pupils may come to see specific examples (single entities) as generic examples (representatives of a class of examples), which in turn help in the abstraction of the general concept".

The computer must show the processes of mathematics as well as giving the final results of any calculation. Often, the process of calculation in a computer programme remains hidden and only the final results are given. Therefore, to help the learner to use the system to the best advantage, and to help in the formation of appropriate concept imagery, an external "organising agent" [16, p. 4] is required, in the shape of guidance from a teacher, a text-book or appropriate teaching material.

In the rest of this article, we shall describe a didactic approach for analysis and reflection relevant to teaching mathematics with computers, taken from our own research observations.

\section{Two case studies}

\subsection{Method}

\subsubsection{Purpose}

The purpose was to contribute to the study of the impact of using mathematics computer software applied to particular situations (graph oriented problems), more specifically to investigate how the use of the software adds to the improvement of students' mathematical achievements.

\subsubsection{Subjects}

In these studies two students, Gabriella and Marianna of 16 participated. They were not very good at mathematics, however, both of them were very hardworking students, eager to improve their mathematical skills and to co-operate 
with their teacher researcher. Both students were taking a second year mathematics course comprising functions, quadratic equations and inequalities etc. The students were generally quite familiar with computers and needed little introduction to the general use of the software.

The research was carried out between April and June, 2003 at a Hungarian Secondary Grammar School (Ady Endre Gimnázium in Debrecen) known to be the third most successful grammar school in Debrecen.

\subsubsection{Measures}

Before students were introduced to the software GRAPHMATICA they were asked to complete a pretest (5 mathematical problems, 100 points) that were designed to measure three areas of mathematical knowledge: transformations of functions, solving quadratic equations and inequalities. After the pretest students participated in a session of 10 mathematics lessons in the computer laboratory where problems in the pretest and other relevant exercises were solved and discussed together with the teacher. During these activities with software an internal methodology based on our observations and analysis of the results of the pretest was used.

Students worked independently with the software but before students switched on their computers a short discussion was presented by the teacher researcher about the mathematical exercises posed to solve, unclear wording, or other issues that might cause students problems as they worked through the software.

Gabriella and her lab partner Maria were discussing the data they collected on transformations of graphs, solutions of quadratic equations and inequalities. Maria's attitude towards the project was "just do enough to get by" while Gabriella was concerned about their conclusions.

During the session of the extra lessons these students went through the rules of transformation of functions ("To which directions should the graph of functions be shifted under certain transformations"), the application of the formula ("What is the relation between the zeros of quadratic functions, the formula and the solution of the quadratic equation") and they practiced to solve quadratic inequalities containing quadratic expressions in their numerator or denominator ("How to study the numerator and the denominator separately then how to find the common intervals for the final solution). Students were free to use computersand the software GRAPHMATICA to solve these graph-oriented problems 


$$
\text { "gyongyosi" — 2004/7/23 — 13:57 — page } 22 \text { — \#8 }
$$

throughout the session.

It was not expected that the students would have full or complete answers to the questions, but having them write their preliminary ideas allowed them the opportunity to compare their initial ideas to their final answers. Students then have the opportunity to present their solutions and check their answers. Whenever they had problems in using the computer or understanding a mathematical concept the teacher gave short hints or interesting related questions that helped to link concepts such as "sketch the function with the help of the computer, make sure to switch the graph of it in the right direction".

Students then continued with challenges two and three in a similar manner starting with solving quadratic equations, again involving computer usage, and then giving solutions for quadratic inequalities.

After this session of 10 lessons they responded to the questions of a posttest. By analysing the students' results and comparing them in the pre- and posttest it can be seen that some considerable developments in the thinking process have appeared that were not revealed from the beginning. In particular, we noted that the use of the software have a profound effect on the improvement of solving quadratic inequalities.

We give a brief description of the construction of the test items and how they fit into this methodology below, in order to understand students' difficulties and how they faced them. This methodology provides us an overview of the extent to which some mathematical software can be effectively applied to achieve improvement of some mathematical skills. However, this instrumental methodology has the disadvantage of remaining extremely local, still we want to draw attention to the importance of integrating computer-based work into certain fields of mathematical curriculum.

\subsection{Pretest}

In the first problem of the pretest students had to plot the functions $y=$ $x^{2} / 2+5$ (8 points) and $y=-3(x-5)^{2}+1 / 2$ (8 points).

As students were expected to have difficulties in plotting functions by using transformations this problem was proposed to review and improve their skills. The computer software provided a colorful and enhancing learning environment to visualise graphs of functions. Practical experience was seen to provide more effective learning and it is easier to remember things after having done them and seen them, rather than merely having read them [16]. 
In the first part of problem 1, two activities: function transformations such as $y_{1}=x^{2} ; y_{2}=x^{2} / 2 ; y_{3}=x^{2} / 2+5$ and plotting these functions with pen and paper were required.

Marianna (Student 1) was not very good at applying transformations to the graph of functions.

Getting started. Beginning work on plotting the basic quadratic function $x^{2}$ should be easy. The teacher has just demonstrated and explained what is wanted and now the student has to carry out transformations. This routine was well practiced, occurring on a daily basis. Marianna sat, doing nothing until the teacher urged her to solve the problem. She simply did not know where to begin.

It seems that getting-started difficulties are not uncommon. They explain why some students appear to be day dreaming or otherwise inattentive. Some attention difficulties may best be considered organizational problems: in a relatively unstructured situation the pupil is not able to start up and organise problemsolving efforts. Since the behaviour does not take place, the pupil seems to be inattentive. Sometimes, getting-started difficulties may be overcome by explicit instructions. Thus Marianna was told to consider what kind of vertical and horizontal shifts and stretching and reflecting should be made. Then Marianna started to plot the transformed functions, however, she did not carry out transformations in the correct order and that led to a wrong solution. She wrote transformations correctly but not practically to plot functions faultlessly. In part a) of the first problem she wrote: $y_{1}=x^{2} ; y_{2}=x^{2}+5 ; y^{3}=x^{2} / 2+5$. Although in the last step instead of $y_{3}=x^{2} / 2+5$ she plotted $y_{3}=\left(x^{2}+5\right) / 2$. In part b) Marianna shifted the graph of the basic function to the wrong direction as she found wrong analogy between the negative sign inside the brackets $(x-5)^{2}$ and shifting the graph to the negative direction, that is to the left by 5 units.

Marianna had only instrumental understanding as she could write steps of transformations correctly, however, she did not have relational understanding as she could not see relations between the syntactic level of assignment rule symbols and the level of transforming the corresponding graphs of functions. Therefore I gave her 6 points out of $16(37.5 \%)$.

Gabriella (Student 2) had no difficulties in plotting functions and transforming their graphs. She solved the first problem perfectly well therefore she got 16 points $(100 \%)$.

In the second problem: students had to solve the equation: $\sqrt{x-1}=-x+3$. This problem was proposed in order to examine which solving method students 
prefer. According to the expectations students preferred the algebraic method to the graphical one.

Using the algebraic method it is important to decide under what conditions the expression on the left-hand side of the particular equation exists. Then one can take the square of the expressions on both sides of the equation and finally one must not forget to compare results with the conditions. In this problem all these complications can be avoided by solving the equation graphically.

In the second problem Marianna was given to solve the equation $\sqrt{x-1}=$ $-x+3$. She started to take the square of both sides. Unfortunately, Marianna could not take the square of the sum of two terms correctly. She made the following mistakes: $(-x+3)^{2}=-x^{2}+9$. Finally, she obtained a quadratic equation which she could solve correctly by using the formula. I appreciated her efforts as 2 points out of 18 (11.11\%).

Gabriella made only a miscalculation by an oversight. Therefore she got 13 points $(72.22 \%)$ for her solution of the second problem.

In the third problem students were expected to use the formula for solving the equations: $0=x^{2}-4 x+9$ and $0=-x^{2}-6 x-13$. These quadratic equations were selected such that there should be negative numbers under the root sign in the formula for solving them. Against expectations it was not the negative number under the square root sign that confused the students.

In the third problem Marianna had similar difficulties with the sign of expressions as in the second exercise. She calculated as $-4^{2}=-16$ and $-6^{2}=-36$. Therefore she obtained only half of the maximum points 8 points out of $16(50 \%)$. While Gabriella solved the third problem perfectly well, she received 16 points $(100 \%)$.

In the fourth problem students had to solve the inequality $5 x-3 \leq x^{2}+4 x+3$. After rearranging the inequality and reducing the expression on its left-hand side to zero the equation $x^{2}-x+6=0$ was expected to have no roots and therefore the original inequality holds for all $x$ in the domain.

As it was expected students could not give a solution for this problem as after rearranging the inequality to $0 \leq x^{2}-x+6$ and solving the equation $0=x^{2}-x+6$ they could not go on and stopped. If they had been able to link the graph of the function $x^{2}-x+6$ they might have obtained the right solution.

Thus, the teacher researcher realised how important it is to visualise such functions and using computers made it easier and faster.

Both students got 8 points out of $20(40 \%)$. 
In the last problem solving the inequality

$$
\frac{2 x-x^{2}}{2 x^{2}-3 x+7}>0 \text { in the set of integers was expected. }
$$

Students were supposed to examine the numerator and the denominator separately and to conclude in simpler inequalities according to the sign conditions and then to find common intervals and select the final solution.

In the fifth problem Marianna started to consider the numerator and the denominator of the fraction separately. But in the first step she made a mistake because she thought that a fraction is positive if the sign of the numerator and that of the denominator is different. After writing these relations she did not know how to continue. She was given 2 points (6.67\%).

This exercise proved to be too difficult for Gabriella and she did not have the slightest idea how to solve it. She got 0 point $(0 \%)$.

\subsection{Activities with the software}

Sometimes the problem is too difficult. It is meaningless to the students because the basic schemes necessary for comprehension are not present; such students need work at an appropriate level of difficulty. Other students may incorrectly estimate the difficulty of the task and need some help or rather encouragement to see that they do indeed know how to respond. That was the situation in the case of Gabriella and Marianna. During the sessions of extra mathematics lessons they often seemed to have lack of self-assurance. They told me several times: "I am so stupid and bad at mathematic!" They needed lots of encouragement and patiently I kept explaining some solutions to more difficult problems several times. I often told my students: "I am glad that you came to this session and thanks for your enthusiasm." As it was not compulsory to attend these lessons. The computer-based mathematics lessons lessened the tension and the students' fear of mathematics. GraphmaticA, by freeing the students from having to make tiring calculations, helped them to concentrate on the mathematical concepts and their relations without getting lost in the process. Their relational understanding improved considerably after the computer-based lessons and one of the students remarked: "Thus, the roots of quadratic equations are the same as the values calculated by the formula for solving quadratic equations and they are also the same points at which the function (parabola in our case) intersects the $x$-axis, that is the zeros of the function!". She was very satisfied to realise these links between concepts. 
I proposed Marianna to substitute certain values of the variable and to calculate some function values in order to find the right direction in horizontal and vertical shifts while transforming graphs of functions. I also explained that multiplication has priority over addition thus stretching and reflecting precede vertical and horizontal shifts.

Students apparently liked playing with different colours while plotting functions. They tried to have other functions such as the sin, cos, tan, cot, etc. and plotted even if it was not their task. They also liked adding labels and remarks to different objects which also helped their relational understanding and finding reasons for "why" questions. During their experimentations with the computer software they zoomed some parts of functions and used almost all possibilities provided by the software. Computers could visualise various cases that inspired students to make further experimentations. Another advantage of using computers was that students had enough time to recognise problems and to ask questions themselves. Thus, students could progress from knowledge of facts to arrive at more complex conclusions through intellectual activity. Each of their questions was answered immediately and their mistakes were corrected and explanations and feedback were given at once. It was a good way to individualise mathematics teaching. There were problems that were understood by only one of the students and she helped the teacher to explain them to her fellow. Hence, students co-operated and worked collectively. For example Gabriella was better at function transformations and she helped Marianna and Marianna helped Gabriella to handle the computer.

\subsection{Posttest}

The first problem in the posttest was to plot the functions $y=-2 x^{2}+5$ and $y=-(x-5) / 3+3$.

Both Marianna and Gabriella solved the first problem perfectly well so they were given 16 points (100\%).

In the second problem students were asked to solve the equation $\sqrt{2-x}=$ $-3+\sqrt{x+7}$ in the set of natural numbers.

Marianna still could not take the square of a two term expression. Therefore she got 2 points out of $18(11.11 \%)$. Gabriella made a sign mistake such as: $\left[-\sqrt{36(x+7)}^{2}=-[36(x+7)]\right.$. She was given 15 points $(83.33 \%)$.

In the third problem they had to solve the following equations $0=x^{2}-x-6$ and $0=-(x+3)^{2}-4$. 
In the third problem Marianna made a sign mistake such as: $-(x+3)^{2}-4=$ $x^{2}+6 x+5$. Thus she got 14 points out of $16(77.78 \%)$. Gabriella miscalculated something: $-9-4=-15$ instead of -13 . She was given 15 points $(93.75 \%)$. Unfortunately, a possible disadvantage of the regular use of computers is that students may get used to carrying out simple calculations with calculators or computers and thus they waste precious time or make syntax errors in typing numbers and operation signs or simply they become incapable to do a sum in their head.

In the fourth problem the inequality $x-3 \geq x^{2}+4 x-6$ was asked to be solved. Both students gave perfect solution and they tended to solve the problem graphically. Both of them were given 20 points (100\%).

In the fifth problem the inequality

$$
\frac{3 x^{2}-7 x+2}{x-1}>0
$$

was posed to solve.

Both students could study the numerator and the denominator separately, however, they had difficulties in finding the common intervals in each case and also in the final solution. Therefore they got 27 points out of 30 (90\%) for their solutions which were almost perfect.

In the following figures results of the pretest and the posttest of the two chosen students can be seen. In pursuing goals of more effective teaching another issue for future monitoring arises from the current work.

\section{Student 1}

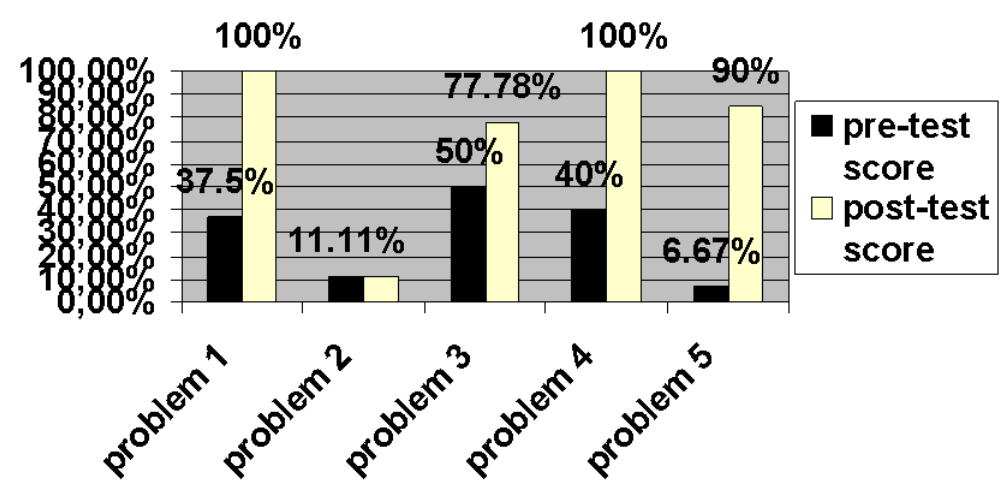




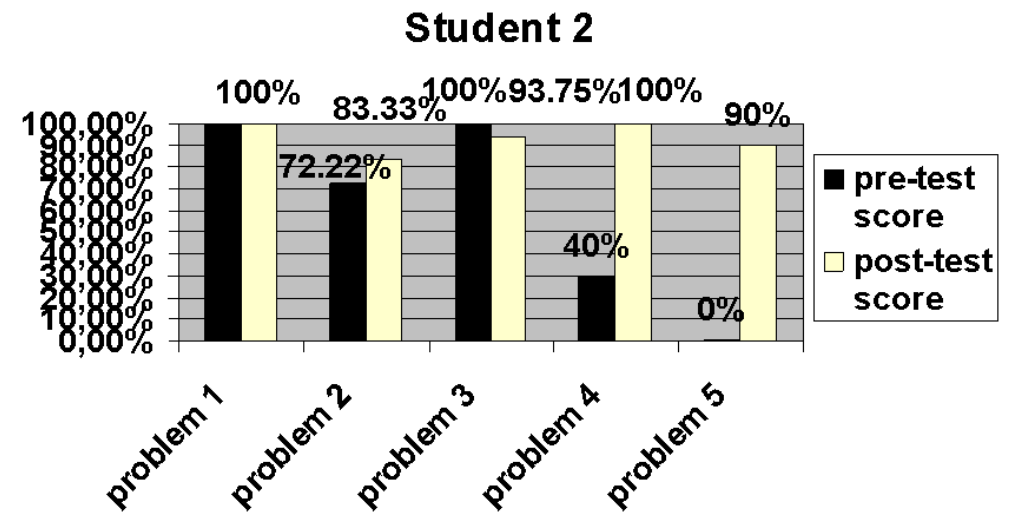

\section{Didactical reflections}

We have carried out research to determine more precisely the impact of a computer-based teaching programme in some graph-oriented problems. This was in response to issues such as reports of studies in which causal aspects of positive outcomes are difficult to target (e.g., Mackie, [9] and the uncritical acceptance of technology encapsulated by Kaput [6]. We have grasped the nettle to try to understand some aspects of what it is that technology brings to teaching and learning mathematics. We showed how computer influence the improvement of two students on some graph-oriented problems.

Test results show that with the computer programme Graphmatica the ability of plotting functions and carrying out function transformations was considerably improved in the case of the first student (from $37.5 \%$ up to $100 \%$ ). Furthermore, in both cases an obvious improvement of the aesthetic sense of the students drawing colorful figures of functions in the posttest can be observed.

In the second problem students tended to choose an algebraic approach while solving the equation and thus the computer software was not useful in this case and it had not much impact on the improvement of their problem-solving.

In the third problem Student 1 had similar problems with the sign of expressions just like in the second problem, however, a slight improvement $30 \%$ can be observed and Student 2 made a small mistake in her calculations therefore her result got worse a little bit, by $5 \%$.

In both cases the greatest improvement can be observed in students' strategies for solving quadratic inequalities. In the simpler case (see problem 4) the improvement of both Student 1 and Student 2 is $60 \%$. 
Although in problem 5 in which students had to solve an inequality being in a form of a fraction with a quadratic expression in the numerator, the use of the computer proved to have a profound effect on the improvement of the students. In the pretest they did not have a clue how to start to solve a problem like this. After using the programme Graphmatica they could better combine the graphical and algebraic approach. In the case of Student 1 the improvement was $83.33 \%$ and in the case of Student 2 it was $90 \%$.

Pedagogies for such instructional sessions are still in the process of development or refinement, and within this enterprise the interaction between mathematics and technology is of considerable importance.

\section{The future}

Our experience shows that the computer can be used as a powerful adjunct to graph-oriented questions.

A future study that looks at the correlation between the usage of mathematical software and algebraic skills such as exploring translations and reflections of graphs and developing graphical approaches to solving equations and inequalities as well as exploring areas of mathematical knowledge in which using computers does not make any positive impact on the improvement of mathematical skills would be of interest.

\section{Appendix}

\section{Pretest}

Problem 1. Plot the following functions:
a) $y=x^{2} / 2+5$,
(8 points)
b) $y=-3(x-5)^{2}+1 / 2$.
(8 points)

Problem 2. Solve the equation:

$$
\sqrt{x-1}=-x+3 . \quad \text { (18 points) }
$$

Problem 3. Solve the following equations:
a) $0=x^{2}-4 x+9$,
(8 points)
b) $0=-x^{2}-6 x-13$.
(8 points) 


$$
\text { "gyongyosi" — 2004/7/23 - 13:57 — page } 30-\# 16
$$

Problem 4. Solve the inequality:

$$
5 x-3 \leq x^{2}+4 x+3 .
$$

(20 points)

Problem 5. Solve the inequality in the set of integers:

$$
\frac{2 x-x^{2}}{2 x^{2}-3 x+7}>0 . \quad \text { (30 points) }
$$

\section{Posttest}

Problem 1. Plot the following functions:
c) $y=-2 x^{2}+5$,
(8 points)
d) $y=-(x-5)^{2} / 3+3$.
(8 points)

Problem 2. Solve the equation in the set of natural numbers:

$$
\sqrt{2-x}=-3+\sqrt{x+7} \quad \quad \text { (18 points) }
$$

Problem 3. Solve the following equations:
c) $0=x^{2}-x-6$,
(8 points)
d) $0=-(x+3)^{2}-4$.
(8 points)

Problem 4. Solve the inequality:

$$
x-3 \geq x^{2}+4 x-6 . \quad \text { (20 points) }
$$

Problem 5. Solve the inequality in the set of positive numbers:

$$
\frac{3 x^{2}-7 x+2}{x-1}>0 . \quad \text { (30 points) }
$$

\section{Acknowledgements}

The author wishes to express here her sincere gratitude to the referees for the many helpful suggestions that were given during this study. 


\section{References}

[1] M. Artigue, J. P. Drouhard and J. B. Lagrange, Acquisition de connaissances concernant l'impact de l'intégration de logiciels de calcul formel dans l'enseignement des mathématiques sur les représentations et pratiques mathématiques des éleves de l'enseignement secondaire, Vol. 7, IREM, Université Paris.

[2] E. Dubinsky, Towards a Theory of Learning Advanced Mathematical Concepts, In: Abstracts of Plenary Lectures and Regular Lectures, ICME9, Tokyo/Makuhari, Japan, 2000.

[3] E. M. Gray and D. O. Tall, Duality, Ambiguity and Flexibility in Successful Mathematical Thinking, In: Proceedings of PME 15, Vol. II, Assisi, Italy, 1991, 72-79.

[4] E. M. Gray and D. O. Tall, Duality, Ambiguity and Flexibility: A Proceptual View of Simple Arithmetic, Journal for Research in Mathematics Education 26 (1994), $115-141$.

[5] E. Gyöngyösi, Continuing Education for Mathematics Teachers of Secondary Education to Use Computers More Effectively and to Improve Education, International Journal for Mathematics Teaching and Learning (2002).

[6] J. J. Kaput and P. W. Thompson, Technology and Mathematics Education, Handbook of Research on Mathematics Teaching and Learning, (In D. A. Grouws, ed.), New York: Macmillan, 1992, 515-556.

[7] J. J. Kaput, Technology in Mathematics Education Research: The first 25 years in the JRME, Journal of Research into Mathematics Education 25(6) (1994), 676-684.

[8] B. Kutzler, 'DERIV(E)-ons vers le future de l'enseignement des mathématiques', Actes de l'Université d'Été: "Les outils de calcul formel dans l'enseignement des mathématiques", (Juge, G., ed.), 1994, 67-68, IREM de Caen.

[9] D. M. Mackie, An evaluation of computer-assisted learning in mathematics, International Journal of Mathematical Education in Science and Technology 23(5) (1992), 731-737.

[10] H. Meissner, Procepts in Geometry, European Research in Mathematics Education 11 (2003).

[11] J. Piaget, The Equilibrium of Cognitive Structures, Harvard University Press, Cambridge MA, 1985.

[12] A. Sfard, Two conceptions of mathematical notions: operational and structural, In: Proceedings of PME-XI, vol. III, Momtréál, Canada, 1987, 162-169.

[13] A. Sfard, Operational origins of mathematical objects and the quandary of reification - the case of function, The Concept of Function: Aspects of Epistemology and Pedagogy, MAA Notes, vol. 25, (G. Harel and E. Dubinszky, eds.), Mathematical Association of America, Washington DC., 1992, 59-84.

[14] O. Tall(ed.), Advanced Mathematical Thinking, Kluwer Academic Publishers, Dordrecht, NL., 1991. 
$\bigoplus$

$$
\text { "gyongyosi" — 2004/7/23 - 13:57 — page } 32-\# 18
$$

[15] D. O. Tall, M. Thomas, G. Davis, E. M. Gray and A. Simpson, What is the object of the encapsulation of a process?, To appear in: Journal of Mathematical Behavior, 2000.

[16] D. O. Tall, 'Building and Testing a Cognitive Approach to the Calculus Using Interactive Computer Graphics', Doctoral Thesis, University of Warwick, 1986.

ERIKA GYÖNGYÖSI

PH.D. STUDENT IN MATHEMATICS-DIDACTICS

UNIVERSITY OF DEBRECEN

H-4010 P.O. BOX 12

HUNGARY

E-mail: gyerika@hotmail.com

(Received August, 2003) 